\title{
Survey of Registered Nurses' Selection of Compression Systems for the Treatment of Venous Leg Ulcers in the UK
}

\author{
Angela Oates ${ }^{\mathrm{a} *}$ and Una Adderley ${ }^{\mathrm{b}}$ \\ ${ }^{\mathrm{a}}$ Centre for Atherothrombosis and Metabolic Disease, Hull York Medical School, Faculty of Health \\ Sciences, University of Hull, Hull, UK. \\ ${ }^{b}$ School of Healthcare, Faculty of Medicine and Health, The University of Leeds, Leeds, U.K.
}

(C) 2019. This manuscript version is made available under the CC-BY-NC-ND 4.0 license

http://creativecommons.org/licenses/by-nc-nd/4.0/

*Corresponding author: Angela Oates, Centre for Atherothrombosis and Metabolic Disease, Hull York Medical School, Faculty of Health Sciences, University of Hull, Hull, UK;

Email:angela.oates@hyms.ac.uk 


\begin{abstract}
Background: Venous leg ulceration is common in older adults in the United Kingdom. The gold-standard treatment is compression therapy. There are several compression bandage and hosiery systems that can be prescribed or purchased, but it was unclear what types of compression systems are currently being used to treat venous leg ulceration within the UK. This online scoping survey of registered nurses sought to (1) to identify what compression systems are available across the UK, (2) how frequently these are in use and (3) if there are any restrictions on their use.

Results: The results showed that registered nurses who treat patients with venous leg ulceration use a wide range of compression systems. The most frequently used systems are the 'less bulky' two-layer elastic and inelastic compression bandaging systems whilst two-layer hosiery was used less frequently and four-layer bandaging used infrequently. Nurses report that certain compression systems are less accessible through the usual procurement routes but this appears to be related to concerns about competency in application techniques.

Conclusions: The data in this survey provides some important insights into the issues around the use of compression therapy for venous leg ulceration in the UK. Limiting access to certain types of compression may promote patient safety but limit patient choice. There may be underuse of the types of compression that promote patient independence, such as hosiery, and over-use of potentially sub-therapeutic therapy such as 'reduced compression'. Overall, this study suggests that further consideration is needed about the provision of compression therapy to UK patients with venous leg ulceration to optimise care and patient choice.
\end{abstract}

\title{
Highlights
}

- In the UK a wide range of compression systems are used to treat venous leg ulceration.

- Nurses report that two-layer bandaging systems are the most frequently used type of system.

- There may be underuse of compression systems that promote patient independence (e.g. two-layer hosiery).

- Sub-therapeutic compression ('reduced compression') may be over-used.

- Four-layer bandaging is used infrequently.

- There are some restrictions on access to certain compression system due to concerns about competency in application techniques.

\section{Keywords}

Bandages, community health nursing, leg ulcer, research, varicose ulcer, wound healing. 


\section{Introduction}

Venous leg ulceration is estimated to affect many older adults in the United Kingdom. It is related to decreased health-related quality of life (Briggs and Flemming, 2007) and incurs significant health costs. A retrospective cohort study estimated that in 2012-13, 278,000 patients received National Health Service (NHS) care for venous leg ulceration which incurred an estimated cost of $£ 941.1$ million per annum (Guest et al., 2018). Another recent study found a community point prevalence rate of 16.4 patients with chronic wounds per 10,000 population of which $25 \%$ had venous leg ulceration (Gray et al., 2018). Both these studies indicate that leg ulceration, and venous leg ulceration in particular, affects a significant proportion of older adults in the UK. Vascular assessment of arterial insufficiency (usually using Doppler-aided ABPI) is an essential part of leg ulcer assessment (Weller et al., 2018). As a significant number of patients with a leg ulcer had not received a Doppler-aided ABPI, it is likely that the incidence of venous leg ulceration is even higher than reported in these studies. The predicted increase in the elderly population, means that the prevalence of venous leg ulceration is likely to rise further, incurring even greater healthcare spend.

Venous leg ulceration is caused by damage to the venous system which disrupts normal blood flow to the tissues, leading to damage and ulcer development. The gold standard therapeutic approach for venous leg ulceration is compression therapy (O'Meara et al., 2012). Compression therapy is the application of external compression and is usually achieved by the application of compression bandaging or hosiery which applies greater pressure at the ankle graduating to less at the knee.

Compression therapy can be delivered by non-elastic and elastic systems (O’Meara et al 2012). Non-elastic compression can be applied using short stretch bandaging systems which provides greater compression at the ankle than at the knee (Nelson and Adderley 2016). Elastic compression can be delivered in the form of multi-layer bandaging systems or two-layer hosiery which are mostly designed to provide $40 \mathrm{mmHg}$ at the ankle graduating to less up the leg (Ashby et al 2014). Elastic hosiery systems have the advantage of being more easily removable and reapplied by patient and carers (Ashby et al 2014). The newer 'Velcro’ systems also allow easier patient/carer removal and reapplication but their efficacy for healing venous leg ulceration is currently unknown. Elastic systems are also available to provide 'reduced' compression to limbs where concomitant arterial impairment is an issue or for patients who are unable to tolerate the recommended dose of $40 \mathrm{mmHg}$. 
The evidence suggests that these different forms of therapy achieve similar therapeutic benefits. Multi-component systems have been found to be more effective than single-component systems but four-layer bandaging and two-component systems appear to be equivalent in terms of healing (O'Meara et al., 2012, Nelson and Adderley, 2016). Four-layer bandaging and high compression hosiery appears to achieve slightly faster healing than short-stretch bandaging (O'Meara et al., 2012). Four-layer bandaging was also found to be more cost-effective than short stretch bandaging but a more recent trial (Ashby et al., 2014) found no difference in rates of venous ulcer healing between four-layer and two-layer, graduated high compression hosiery. However, hosiery was slightly more cost effective and associated with slightly higher quality of life. The results from three small randomised controlled trials (Moffatt et al., 2008, Lazareth et al., 2012, Szewczyk et al., 2010) suggest that two-layer compression bandaging systems may have similar effectiveness to four-layer bandaging but these results are uncertain due to methodological issues. A relatively recent addition to the toolbox of compression are Velcro wrap devices which have been previously used to treat lymphoedema. They may offer an additional form of compression for venous leg ulceration but their clinical and costeffectiveness is currently unknown in relation to healing venous leg ulceration.

Given the strength of the evidence in favour of compression bandaging and hosiery, and the high levels of compression use achieved in older audits (Royal College of Nursing, 2008) it would be reasonable to expect similarly levels of use in current practice. However, recent evidence suggests that too few patients with venous leg ulceration are receiving compression (Guest et al 2018). A recent cross-sectional survey of eight community services in five Northern England NHS Trusts found that 31\% of patients diagnosed with venous leg ulceration were receiving no compression at all. There were similar results from another study which found that $25 \%$ of patients with venous leg ulceration were not documented as receiving any compression (Guest et al., 2018). Although healing was observed in patients with venous leg ulceration who never received compression, the mean time to healing was significantly longer among this group of patients. Another worrying issue was that a further $22 \%$ were in some form of reduced compression (Gray et al., 2018) i.e. receiving sub-therapeutic levels of compression.

The underuse of compression therapy for venous leg ulceration is a cause for concern and may be linked to problems accessing ABPI assessment, lack of confidence in clinical decision making, patient preferences, clinician preferences or product availability but further investigation is needed. 


\section{AIMS}

The aims of this study were (1) to identify what compression systems are available across the UK for treating venous leg ulceration, (2) how frequently these are in use and (3) if there are any restrictions on their use.

\section{ETHICS}

This study was approved by the University of Leeds, School of Healthcare Research Ethics Committee [Ref no: HREC17-040].

\section{METHODS}

This was an online scoping survey covering the UK. The survey consisted of six questions that were administered using Online Surveys (formally Bristol Online Surveys) (Jisc, Bristol, UK). The survey was anonymous with no personal identification information collected and comprised of broad questions so no individual or practice could be identified from the submitted data. (See Table 1)

\begin{tabular}{|l|l|}
\hline \multicolumn{2}{|l|}{ Table 1: Survey Questions } \\
\hline $\begin{array}{l}\text { 1. Please select a response from the drop } \\
\text { down box: }\end{array}$ & a. I understand the information and want to \\
& bolunteer to complete the survey. \\
& c. I l have read like more information about the \\
& take part in the survey.
\end{tabular}




\begin{tabular}{|c|c|}
\hline $\begin{array}{l}\text { 6. Which of the following compression } \\
\text { systems do you use? }\end{array}$ & $\begin{array}{l}\text { a. Four layer bandaging } \\
\text { b. "Reduced" compression e.g. four layer } \\
\text { reduced to three layer } \\
\text { c. Two layer elastic bandaging } \\
\text { d. Two layer inelastic bandaging (Short stretch } \\
\text { bandaging) } \\
\text { e. Two layer hosiery kits } \\
\text { f. Velcro -fastened compression systems } \\
\text { g. Other (please specify) }\end{array}$ \\
\hline $\begin{array}{l}\text { 7. Please rank these compression systems } \\
\text { according to how frequently you use them. } \\
\text { ( } 1=\text { Frequently, } 2=\text { Occasionally } 3=\text { Rarely } \\
\text { and } 4=\text { Never) }\end{array}$ & $\begin{array}{l}\text { a. Four layer bandaging } \\
\text { b. "Reduced" compression e.g. four layer } \\
\text { reduced to three layer } \\
\text { c. Two layer elastic bandaging } \\
\text { d. Two layer inelastic bandaging (Short stretch } \\
\text { bandaging) } \\
\text { e. Two layer hosiery kits } \\
\text { f. Velcro - fastened compression systems } \\
\text { g. Other (please specify) }\end{array}$ \\
\hline $\begin{array}{l}\text { 8. Are there any compression systems } \\
\text { excluded from use? (e.g. due to restriction } \\
\text { on a local formulary) }\end{array}$ & $\begin{array}{l}\text { 1. Yes (please specify). } \\
\text { 2. No }\end{array}$ \\
\hline
\end{tabular}

The inclusion criteria required participants to be a registered nurse, currently employed in the UK who treated patients with venous leg ulcers. The survey was advertised through non-NHS organisations relevant to treatment of venous leg ulcers such as the Tissue Viability Society, the Lindsay Leg Club and The Leg Ulcer Forum and via social media platforms such as Facebook and Twitter. The Online survey was available for two months between $23^{\text {rd }}$ May 2018 and $23^{\text {rd }}$ July 2018. There was no limitation on the number of participants.

Quantitative data from the survey was analysed with descriptive statistics using Excel (Microsoft Office 2013).

\section{RESULTS}

\section{Participants}

A total of 139 individuals volunteered to complete the survey. One individual bypassed the informed consent page and these responses were removed from the analysis. Of the remaining 138, four read the information and did not want to take part in the survey, two were not registered nurses working with venous leg ulcers and one was not currently employed within the UK. This left a total of 131 eligible participants who completed the survey. The average survey completion time was approximately 4 minutes. Data is presented as percentage of participants responses. 


\section{Care setting}

Of these participants, 58.8\% provided leg ulcer care in England, 37.4\% in Scotland, 3.1\% in Wales and $0.8 \%$ in Northern Ireland.

The participants delivered venous leg ulcer care across multiple care settings, sometimes across several settings. 15.3\% delivered care in GP practices, $68.7 \%$ in district nursing teams, $14.5 \%$ in nursing homes, 32.8\% in leg ulcer/vascular clinics, 13\% in hospital and 3.1\% at Leg Clubs (Figure 1). Of the reported care setting; 10.7\% delivering care in other settings which included; 'tissue viability services and wound clinics', 'acute care within community', 'patient at home' services, prisons, nursing homes, lymphoedema services, plastic surgery out-patient clinics and mental health wards. One participant reported that compression bandaging was not undertaken for in-patients because staff lacked capacity and competence.

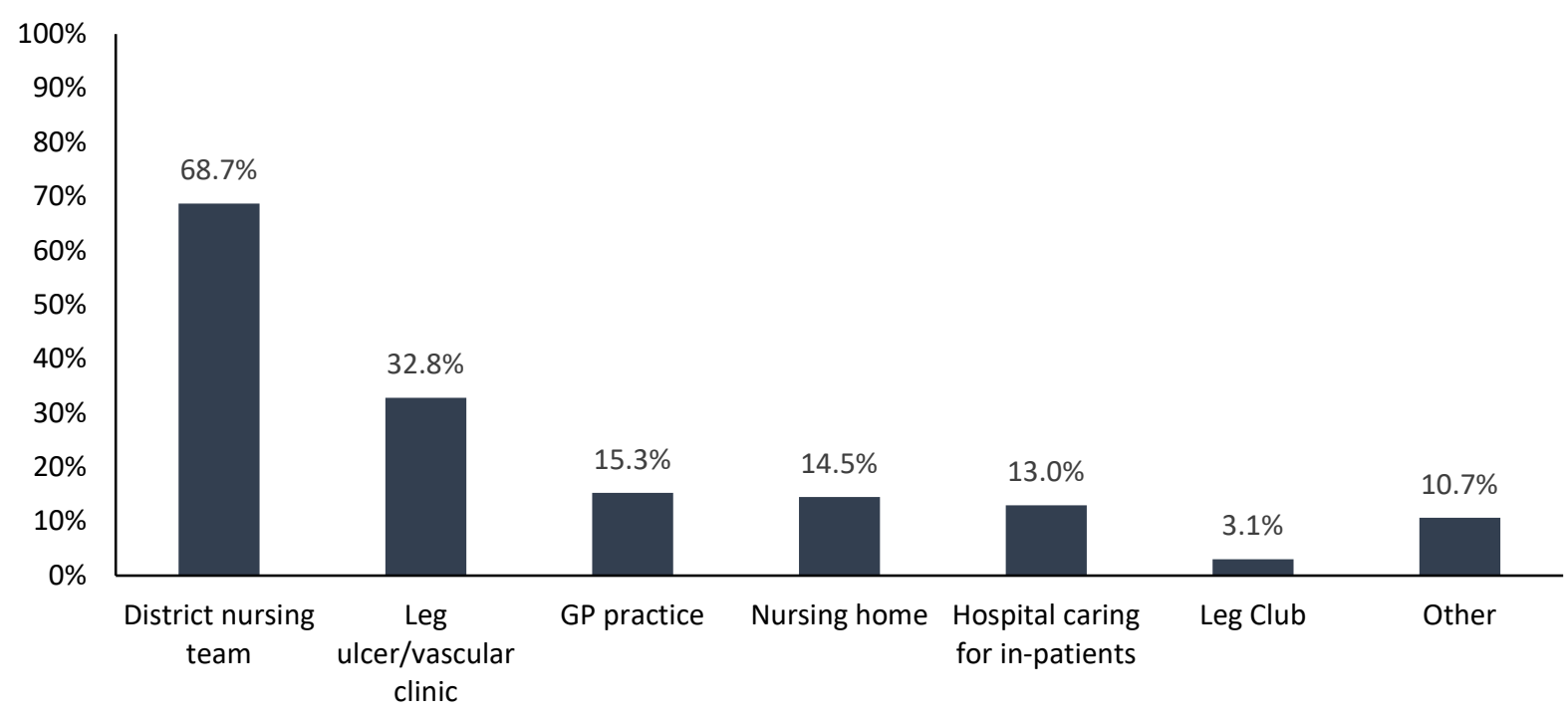

Figure 1. Reported care settings where participants delivered venous leg ulcer care

\section{Which Compression Systems are in use?}

Between $18.3 \%$ - 44.3\% of participants use each of the different compression systems listed in the survey. Of the elastic compression systems; $62.9 \%$ of the participants used four-layer bandaging, 71\% used two-layer elastic bandaging and 78.2\% used two-layer hosiery kits. For 
inelastic compression; 76.6\% used two-layer inelastic bandaging. The use of Velcro-fastened compression systems was reported by $73.4 \%$ of participants and $71 \%$ reported using 'reduced' compression which included the use of 'reduced compression kits.

Other compressions systems reported (4\%) included flat knit hosiery, "stocking liners only" (for patients could not tolerate full compression) and "bespoke compression regimes" (it was unclear what these consisted of).

\section{Which compression systems are used most frequently?}

Figure 2 shows how frequently participants used the various compression systems to treat VLU. The most frequently used compression systems were those that involved fewer layers. Two-layer inelastic (short stretch) bandaging and two-layer elastic bandaging was frequently used by $53.4 \%$ and $51.2 \%$ of participants respectively (although $18.3 \%$ reported that they never use 2-layer inelastic (short stretch) bandaging and a further $11.5 \%$ reported that they never use 2-layer elastic bandaging).

Two-layer hosiery kits were frequently used by only $36.7 \%$ of participants. Only $16.8 \%$ of participants reported frequent use of the bulkier four-layer bandaging systems. $19.08 \%$ of participants reported that they frequently used Velcro -fastened compression systems. 30\% of participants reported that they frequently used reduced compression. 


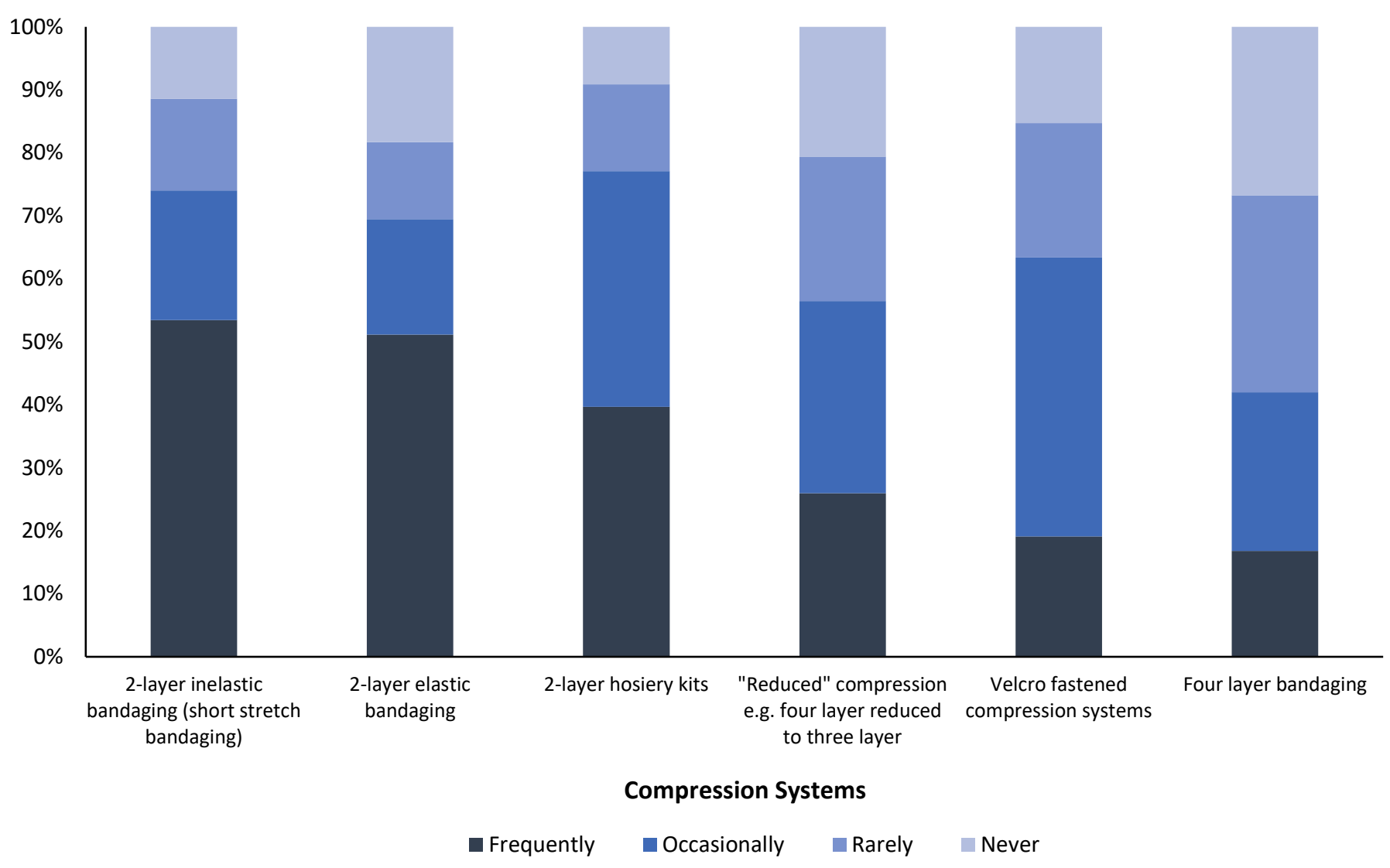

Figure 2. Participants’ frequency of use of various compression systems

\section{Reported Restrictions for Use of Compression Systems}

43 participants (32.8\%) reported that they were unable to use some compression systems. Six participants (14\%) attributed restrictions to formulary rationalisation (i.e a range of types of compression were available but only particular makes could be accessed). Nine participants (21\%) reported that they had no access to 4-layer bandaging systems. Nine participants (21\%) reported no access to 2-layer systems. Of these five had no access to 2-layer elastic bandaging systems, two had no access to 2-layer hosiery compression systems and two did not specify which type of '2-layer'. Two participants (4.6\%) reported that there was limited access to compression for generalist clinicians and referral to specialist clinicians was required to access compression. One participant reported difficult in accessing a certain make of compression. Four participants (9.3\%) reported that restrictions on the availability of different bandaging systems were linked with concerns about clinician competency in application technique. One participant reported that they did not have access to Velcro-fastened compression systems. 


\section{DISCUSSION}

Compression therapy is the cornerstone of good quality care for venous leg ulceration. The results of this study would suggest that a wide range of compression systems are being used to care for patients with venous leg ulceration. It is interesting to note however, that the compression systems that are being used most frequently are those with least bulk i.e. the twolayer bandaging and hosiery systems. It is unclear whether this is due to patient preference or whether two-layer systems are easier and quicker for clinicians to apply. It is a matter of concern that two-layer elastic bandaging systems are in such widespread use, despite the lack of robust evidence for their clinical and cost effectiveness. Given patients and clinicians' apparent preference for this type of compression system, there is need to establish the relative effectiveness of this type of system compared to other more evidence-based compression systems.

It was also surprising that 'reduced compression' is being used so frequently for patients with venous leg ulceration. A diagnosis of 'venous leg ulceration' indicates that there are no concomitant underlying issues (such as arterial impairment) that might contraindicate 'full' compression therapy of $40 \mathrm{mmHg}$ at the ankle. It is unclear whether the frequent use of reduced compression is being driven by patient preference (which in turn, may be driven by inadequate analgesia to address discomfort and pain) or other factors. Either way, the relatively frequently use of 'reduced compression' suggests sub-optimal therapy for healing venous leg ulceration is not unusual.

This study revealed that local restrictions on the types of compression systems available in different practice areas are not uncommon. These restrictions are reported to be due to concerns about maintaining adequate clinician competency in application for a wide range of different compression systems. Such restrictions are understandable in terms of promoting patient safety and reducing the risks associated with poor competency. However, limiting therapeutic options reduces patient choice and may also lead to sub-optimal care.

The reasons for the practice revealed in this survey are beyond the scope of this study. However, it is worth noting that it has been suggested that barriers exist that impact on the appropriate provision of compression therapy for treating venous ulceration (Franks et al., 2016). Cost of wound care products may be an issue although this is less relevant in the UK where most wound care is provided free of charge to the patient (Franks et al 2016). However, it is possible that despite robust evidence of the cost-effectiveness of compression therapy 
(O’Meara et al 2012) clinicians responsible for delivering care may under-value such health economic data. Excessive workloads, inadequate organisational support and financial pressure may hinder the delivery of adequate compression (Franks et al 2016). Patient related issues may also impact on the choice of compression therapies as indicated by the participants who reported the reluctance of patient to accept 4-layer bandaging. There is limited evidence about patient views on compression (Franks et al 2016) but patients may be influenced by nurses' belief in the effectiveness of compression (Jull et al., 2004) and care that pays attention to patients’ preferences and pain issues (Van Hecke et al., 2011).

It can be challenging to identify a compression system that is acceptable to each individual patient. Patients' limbs can greatly vary in terms of length, circumference, severity of wounding, and pain levels. Patients are also likely to have individual preferences around what is acceptable in terms of the bulk and appearance of a compression system and its impact on footwear and clothing choices. A bulky four-layer system may limit footwear options, but a two-layer hosiery system may be uncomfortable. If clinicians are to meet the individual needs of each patients, they need to be able to offer a wide range of compression therapies and this can only be achieved safely and effectively if they have enough practice opportunities to develop expert knowledge and skills for each type of compression system. This may be difficult to achieve in generalist practice where compression therapy is only one of the many areas of care clinicians are required to deliver.

One solution may be to use compression therapies that allow patients greater independence and autonomy in applying their own compression. There is good evidence that two-layer hosiery is both clinically and cost effective for those patients who can tolerate it (Ashby et al 2014). It is unclear whether the relatively infrequent use of two-layer hosiery systems shown in this study is due to patient preference or clinician preference. Velcro-fastened systems may offer similar benefits re patient independence but at present their clinical effectiveness for promoting healing of venous leg ulceration is unknown.

\section{Strengths and Limitations}

The sample provides a current insight into the use of compression systems in the UK for venous leg ulceration but there are some limitations. Although this study achieved a reasonable number of responses, the use of convenience sampling meant the sample may not be representative of UK practice in general. As this was an anonymised survey, we were unable to conduct any data validation, and thus unable to verify whether the participants were registered nurses with 
recent experience of caring for patients with venous leg ulceration. It is also worth noting that the reported data is the participants' view of their current practice area which may be different to the actual compression prescribing in their practice area.

\section{Conclusions}

The data in this survey provides some important insights into the issues around the use of compression therapy for venous leg ulceration in the UK. Compression selection appears more driven by the wish for reduced bulk than by research evidence. Limiting access to certain types of compression may promote patient safety but limit patient choice. There may be underuse of the types of compression, such as hosiery, that promote patient independence and over-use of 'reduced compression' that is likely to offer sub-therapeutic care. Overall, this study suggests that further consideration should be given to how compression therapy is provided to UK patients to optimise care and patient choice. 


\section{REFERENCES}

ASHBY, R. L., GABE, R., ALI, S., SARAMAGO, P., CHUANG, L. H., ADDERLEY, U., BLAND, J. M., CULLUM, N. A., DUMVILLE, J. C., IGLESIAS, C. P., KANG'OMBE, A. R., SOARES M.O, STUBBS, N. C. \& TORGERSON, D. J. 2014. VenUS IV (Venous leg Ulcer Study IV) - compression hosiery compared with compression bandaging in the treatment of venous leg ulcers: a randomised controlled trial, mixed-treatment comparison and decision-analytic model. Health Technology Assessment.

BRIGGS, M. \& FLEMMING, K. 2007. Living with leg ulceration: a synthesis of qualitative research. Journal of Advanced Nursing, 319-328.

FRANKS, P. J., BARKER, J., COLLIER, M., GETHIN, G., HAESLER, E., JAWIEN, A., LAEUCHLI, S., MOSTI, G., PROBST, S. \& WELLER, C. 2016. Management of Patients With Venous Leg Ulcers: Challenges and Current Best Practice. J Wound Care, 25 Suppl 6, S1-s67.

GRAY, T. A., RHODES, S., ATKINSON, R. A., ROTHWELL, K., WILSON, P., DUMVILLE, J. C. \& CULLUM, N. A. 2018. Opportunities for better value wound care: a multiservice, cross-sectional survey of complex wounds and their care in a UK community population. BMJ Open, 8.

GUEST, J. F., FULLER, G. W. \& VOWDEN, P. 2018. Venous leg ulcer management in clinical practice in the UK: costs and outcomes. International Wound Journal, 15, 29-37.

JULL, A. B., MITCHELL, N., ARROLL, J., JONES, M., WATERS, J., LATTA, A., WALKER, N. \& ARROLL, B. 2004. Factors influencing concordance with compression stockings after venous leg ulcer healing. $J$ Wound Care, 13, 90-2.

LAZARETH, I., MOFFATT, C., DISSEMOND, J., LESNE PADIEU, A. S., TRUCHETET, F., BEISSERT, S., WICKS, G., TILBE, H., SAUVADET, A., BOHBOT, S. \& MEAUME, S. 2012. Efficacy of two compression systems in the management of VLUs: results of a European RCT. $J$ Wound Care, 21, 553-4, 556, 558 passim.

MOFFATT, C. J., EDWARDS, L., COLLIER, M., TREADWELL, T., MILLER, M., SHAFER, L., SIBBALD, G., BRASSARD, A., MCINTOSH, A., REYZELMAN, A., PRICE, P., KRAUS, S. M., WALTERS, S. A. \& HARDING, K. 2008. A randomised controlled 8-week crossover clinical evaluation of the 3M Coban 2 Layer Compression System versus Profore to evaluate the product performance in patients with venous leg ulcers. Int Wound J, 5, 267-79.

NELSON, E. A. \& ADDERLEY, U. 2016. Venous Leg Ulcers. BMJ Clinical Evidence [Online].

O'MEARA, S., CULLUM, N., NELSON, E. A. \& DUMVILLE, J. 2012. Compression for Venous Leg Ulcers. Cochrane Database of Systematic Reviews 2012, Issue 11.

ROYAL COLLEGE OF NURSING 2008. National Audit: the Management of Venous Leg Ulcers. www.rcn-audit.org.uk.

SZEWCZYK, M. T., JAWIEŃ, A., CIERZNIAKOWSKA, K., CWAJDA-BIAŁASIK, J. \& MOŚCICKA, P. 2010. Comparison of the effectiveness of compression stockings and layer compression systems in venous ulceration treatment. Archives of Medical Science : AMS, 6, 793-799.

VAN HECKE, A., VERHAEGHE, S., GRYPDONCK, M., BEELE, H. \& DEFLOOR, T. 2011. Processes underlying adherence to leg ulcer treatment: a qualitative field study. Int J Nurs Stud, 48, $145-55$. 
WELLER, C. D., TEAM, V., IVORY, J. D., CRAWFORD, K. \& GETHIN, G. 2018. ABPI reporting and compression recommendations in global clinical practice guidelines on venous leg ulcer management: A scoping review. Int Wound $J$. 